\title{
Nanoparticulate formulations of mithramycin analogs for enhanced cytotoxicity
}

This article was published in the following Dove Press journal:

International Journal of Nanomedicine

7 November 201I

Number of times this article has been viewed

\section{Daniel Scott \\ Jürgen Rohr \\ Younsoo Bae}

Department of Pharmaceutical Sciences, College of Pharmacy, University of Kentucky, Lexington, KY, USA
Correspondence: Younsoo Bae Department of Pharmaceutical Sciences, College of Pharmacy, University of Kentucky, 789 South Limestone, Lexington, KY 40536-0596, USA

$\mathrm{Tel}+\mathrm{I} 8593236649$

Fax +l 8592577564

Email younsoo.bae@uky.edu
Abstract: Mithramycin (MTM), a natural product of soil bacteria from the Streptomyces genus, displays potent anticancer activity but has been limited clinically by severe side effects and toxicities. Engineering of the MTM biosynthetic pathway has produced the 3-side-chainmodified analogs MTM SK (SK) and MTM SDK (SDK), which have exhibited increased anticancer activity and improved therapeutic index. However, these analogs still suffer from low bioavailability, short plasma retention time, and low tumor accumulation. In an effort to aid with these shortcomings, two nanoparticulate formulations, poly(ethylene glycol)-poly(aspartate hydrazide) self-assembled and cross-linked micelles, were investigated with regard to the ability to load and $\mathrm{pH}$ dependently release the drugs. Micelles were successfully formed with both nanoparticulate formulations of each drug analog, with an average size of $8.36 \pm 3.21$ and $12.19 \pm 2.77 \mathrm{~nm}$ for the SK and SDK micelles and $29.56 \pm 4.67 \mathrm{~nm}$ and $30.48 \pm 7.00 \mathrm{~nm}$ for the SK and SDK cross-linked micelles respectively. All of the drug-loaded formulations showed a $\mathrm{pH}$-dependent release of the drugs, which was accelerated as $\mathrm{pH}$ decreased from 7.4 to 5.0. The micelles retained biological activity of SK and SDK entrapped in the micelles, suppressing human A549 lung cancer cells effectively.

Keywords: drug delivery, polymer micelles, controlled release, MTM, cancer chemotherapy

\section{Introduction}

Mithramycin (MTM), an aureolic acid-type polyketide antibiotic produced naturally by soil bacteria from the Streptomyces genus, exhibits anticancer activity by cross-linking GC-rich DNA and shutting down proto-oncogenes, particularly those triggered by Sp (specificity protein) transcription factors, eg, $\mathrm{Sp} 1$ and $\mathrm{Sp} 3 .{ }^{1-5}$ Overexpression of $\mathrm{Sp} 1$ has been observed in several cancers and has been linked to the control of cell growth, survival, and differentiation, all of which are important in cancer progression. ${ }^{6-8}$ MTM has been used clinically in the past to treat testicular cancer, Paget's bone disease, and hypercalcemia but has been severely limited by its poor bioavailability and toxic side effects such as hepatic, gastrointestinal, bone marrow, and renal toxicities. ${ }^{9-13}$ In order to address these issues, extensive combinatorial biosynthesis has been performed on the MTM pathway and has resulted in several novel analogs. ${ }^{14-16}$ The present authors have shown that the inactivation of the $m t m W$ gene, which codes for the last enzyme in the MTM biosynthetic pathway, yields the most favorable of the new analogs, MTM SK (SK) and MTMSDK (SDK). ${ }^{15,16}$ Both SK and SDK exhibited higher anticancer activity than the native MTM, ${ }^{15,16}$ yet their short plasma retention time and low accumulation in tumors remained to be improved. 
A drug-delivery system is a technique that ensures drug molecules are delivered to disease lesions at the right amount and timing by using drug carriers. Nanoparticles are widely used as drug carriers because large molecules $(>50 \mathrm{kDa})$ can avoid renal clearance and circulate in the body for prolonged time in comparison to small molecules. ${ }^{17}$ Tumors are characterized with leaky blood vessels and immature lymphatic drainage, which allow nanoparticles (20-200 nm) to accumulate in tumor tissues. ${ }^{18}$ Nanoparticles take advantage of both prolonged plasma retention time and preferential tumor accumulation to deliver drug payloads to tumors preferentially. ${ }^{19}$ Liposomes, dendrimers, and polymer micelles are nanoparticulate formulations used widely for tumor-preferential delivery of therapeutic agents. Among these formulations, polymer micelles offer a versatile platform that can be prepared through self-assembling of block copolymers in aqueous solutions..$^{20}$ Self-assembled polymer micelles possess a core compartment enveloped by a hydrophilic shell. The core-shell structure protects drug payloads from precipitation, protein adsorption, and enzymatic degradation in the body. The polymer micelles can dissociate as the core becomes unstable under conditions accompanied by dilution, drug release, or polymer degradation. ${ }^{21}$ Polymer micelles can be stabilized by cross-linking in the core or shell to avoid premature micelle dissociation during tumorpreferential drug delivery. ${ }^{22,23}$

In addition to tumor-preferential drug delivery, controlled drug release is another important factor for nanoparticles to maximize therapeutic efficacy of drug payloads. Drug binding linkers are normally designed to degrade in response to $\mathrm{pH}$, enzymatic activity, light, or heat. ${ }^{24}$ The hydrazone bond, involved in many biomolecular events, is stable at physiological $\mathrm{Ph}$ (7.4), yet is cleaved in acidic conditions $(\mathrm{pH}<7.0)$ in a $\mathrm{pH}-$ dependent manner. Such a unique degradation pattern has been employed to design $\mathrm{pH}$-controlled drug-delivery systems that can trigger drug release in intracellular lysosomal compartments ( $\mathrm{pH} 5.0$ ), following the cellular uptake of drug carriers. ${ }^{25}$ Tumor tissues are also acidic ( $\mathrm{pH}$ 6.5-7.0) due to the inefficient glucose consumption that massively produces lactic acids. ${ }^{26}$ Low $\mathrm{pH}$ in tumors is also attributed to high flux at the glyceraldehydes3-phosphate dehydrogenase step of glycolysis. ${ }^{27}$ These two factors contribute to tumor acidosis in combination. The authors of this paper previously demonstrated that polymer micelles to which drug molecules were conjugated through acid-sensitive hydrazone linkers could accelerate drug release in cancer cells and reduce systemic toxicity in animal tumor models. ${ }^{28}$ The hydrazone is a chemical bond between ketone and hydrazide groups, and thus, drug molecules containing ketone groups are advantageous to design $\mathrm{pH}$-sensitive drugdelivery systems. ${ }^{29-31}$

Figure 1 shows the likely chemical structures of MTM analogs (SK and SDK) and two nanoparticulate formulations (self-assembled and cross-linked polymer micelles) used in this study. SK and SDK possess one and two ketone groups, respectively, in their 3-side chains, plus an additional keto group in the tricyclic ring system. At least one of the ketone groups is subsequently bound to hydrazide groups of poly(ethylene glycol)-poly(aspartate hydrazide) [PEG-p(AspHyd)] block copolymers to prepare drug-conjugated polymer micelles. The acid-labile hydrazone bonds between the block copolymers and MTM analogs are used to accelerate drug release in acidic conditions corresponding to tumor tissues in vivo. Self-assembled and cross-linked micelles are compared to investigate the influence of micelle stability on drugrelease patterns. A549 cell lines (human nonsmall cell lung cancer) are used to test biological activity of nanoparticulate MTM formulations. This article, therefore, provides an insight and understanding of the design, development, and properties of MTM nanoparticulate formulations for effective cancer therapy.

\section{Materials and methods Materials}

L-aspartic acid $\beta$-benzyl ester (BLA), triphosgene, anhydrous hydrazine, $\mathrm{N}, \mathrm{N}^{\prime}$-diisopropylcarbodiimide (DIC), N-hydroxysuccinimide (NHS), anhydrous tetrahydrofuran (THF), anhydrous hexane, anhydrous ethyl ether, anhydrous dimethyl sulfoxide (DMSO), DMSO (molecular biology grade, $\geq 99.9 \%$ ), DMSO-d ${ }_{6}$, resazurin sodium salt, acetate buffer solution, phosphate buffer solution, adipic acid, and sodium hydroxide $(\mathrm{NaOH})$ were purchased from SigmaAldrich (St Louis, MO). $\alpha$-Methoxy- $\omega$-amino PEG (5 kDa) was purchased from NOF Corporation (Tokyo, Japan). Regenerated cellulose dialysis bags with molecular weight cutoff(MWCO) 6-8 kDa and Slide-A-Lyzer G2 dialysis cassettes with MWCO $10 \mathrm{kDa}$, methanol $(\mathrm{MeOH})$, acetonitrile (ACN), celite, C18 RP silica gel, tryptic soy broth (TSB), LB broth, Difco ${ }^{\mathrm{TM}}$ agar, sucrose, potassium sulfate, magnesium chloride, glucose, casamino acids, yeast extract, MOPS (3-morpholinopropane-1-sulfonic acid), and trace elements were purchased from Fisher Scientific (Ottawa, ON). Streptomyces argillaceus ATCC 12956 was purchased from ATCC (Manassas, VA). Spectra/Por 6 dialysis tubing with MWCO $50 \mathrm{kDa}$ was purchased from Spectrum Labs (Fort Lauderdale, FL). 

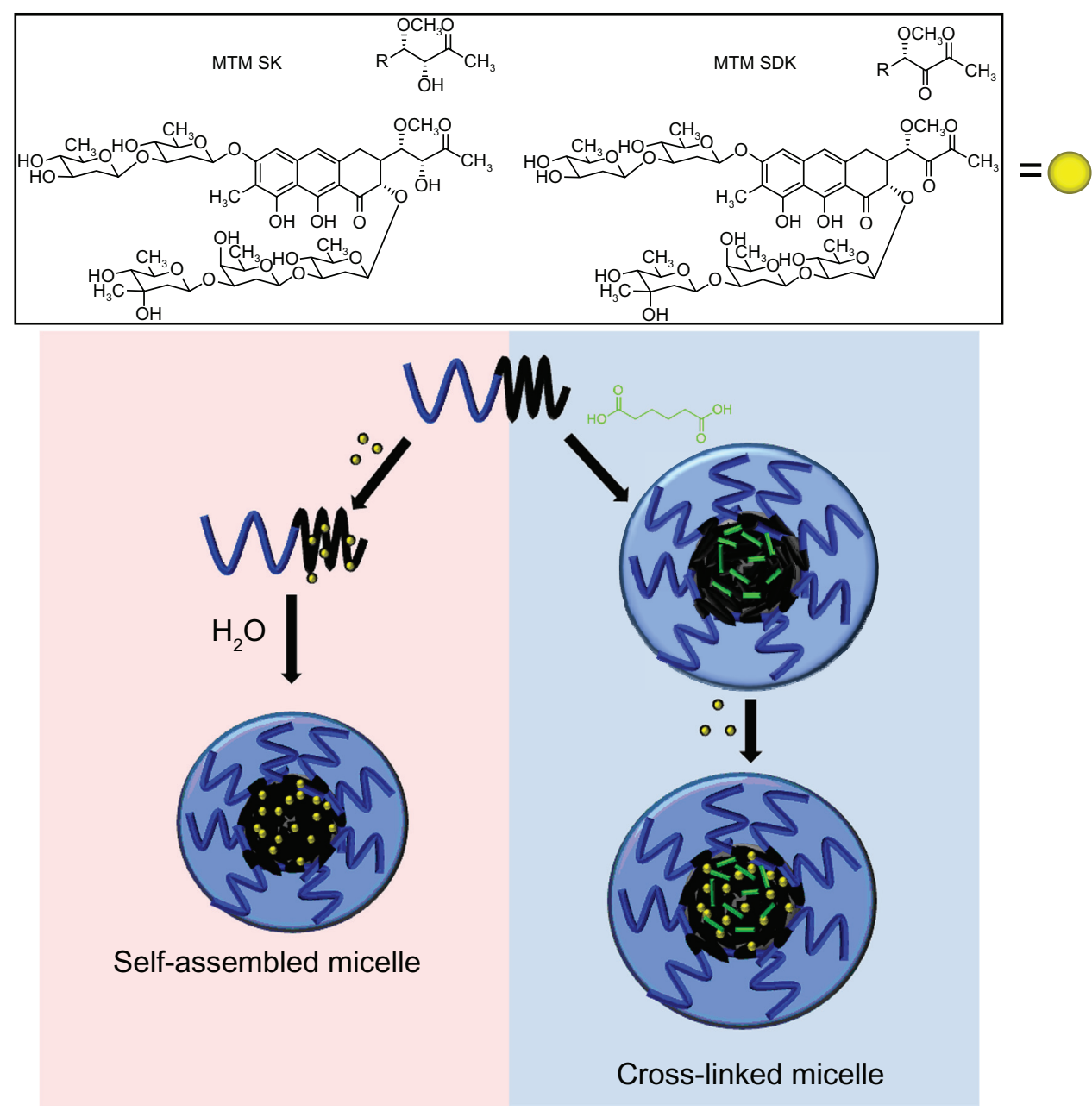

Figure I Self-assembled and cross-linked polymer micelles as nanoparticulate formulations for MTM analogs. Abbreviation: MTM, mithramycin.

\section{Biosynthesis of MTM analogs}

SK and SDK were produced by an adapted procedure reported previously. ${ }^{15}$ Cells of $S$. argillaceus M7W1 were plated on R5A agar and grown for 4 days. After 4 days, the spores were used to inoculate $100 \mathrm{~mL}$ of TSB for 24 hours in an orbital shaker at $28^{\circ} \mathrm{C}, 250 \mathrm{rpm}$. After 24 hours, $4 \mathrm{~mL}$ of the TSB culture was used to inoculate $40,100 \mathrm{~mL}$ flasks of R5A media. The cultures were grown for 3 days at $28^{\circ} \mathrm{C}$, $250 \mathrm{rpm}$. After 3 days the cells were harvested and $50 \mathrm{~g} / \mathrm{L}$ celite was added to the culture. The cells were removed by filtration, re-dissolved in $\mathrm{MeOH}$ and sonicated for 1 hour to lyse the cells. After sonication the cells were filtered again and the $\mathrm{MeOH}$ was removed from the filtrate, reconstituted in water, and loaded onto a $5 \mathrm{~cm} \times 12 \mathrm{~cm} \mathrm{C18} \mathrm{RP} \mathrm{column}$ equilibrated with 10 column volumes of water. The column was washed with $10 \% \mathrm{ACN}$ in water, followed by $20 \% \mathrm{ACN}$ in water, and subsequently eluted with $100 \% \mathrm{ACN}$. The filtrate from the initial removal of the cells was also loaded onto the C18 column, followed by the same fractionation procedure. The samples were dried and re-dissolved in $80 \%$ $\mathrm{MeOH}$ and water. The compounds were further isolated by semi-preparative high-performance liquid chromatography (HPLC).

\section{Block copolymer synthesis}

Block copolymers were synthesized as reported previously (Figure 2). ${ }^{22}$ In brief, BLA-N-carboxy anhydride (BLA-NCA) was prepared as a monomer by adding $2.88 \mathrm{~g}$ of triphosgene to $5.0 \mathrm{~g} \beta$-benzyl-L-aspartate in $100 \mathrm{~mL}$ of dry THF at $45^{\circ} \mathrm{C}$ until the solution became clear. Anhydrous hexane was added to the solution slowly to form BLA-NCA crystal, which was recrystallized at $-20^{\circ} \mathrm{C}$. PEG was used as a macroinitiator to conduct the ring-opening polymerization of BLA-NCA, which produced PEG-( $\beta$-benzyl L-aspartate) [PEG-PBLA]. BLA-NCA and PEG were reacted in anhydrous DMSO at $50 \mathrm{mg} / \mathrm{mL}$ under $\mathrm{N}_{2}$ for 2 days at $45^{\circ} \mathrm{C}$. The PEG-PBLA 


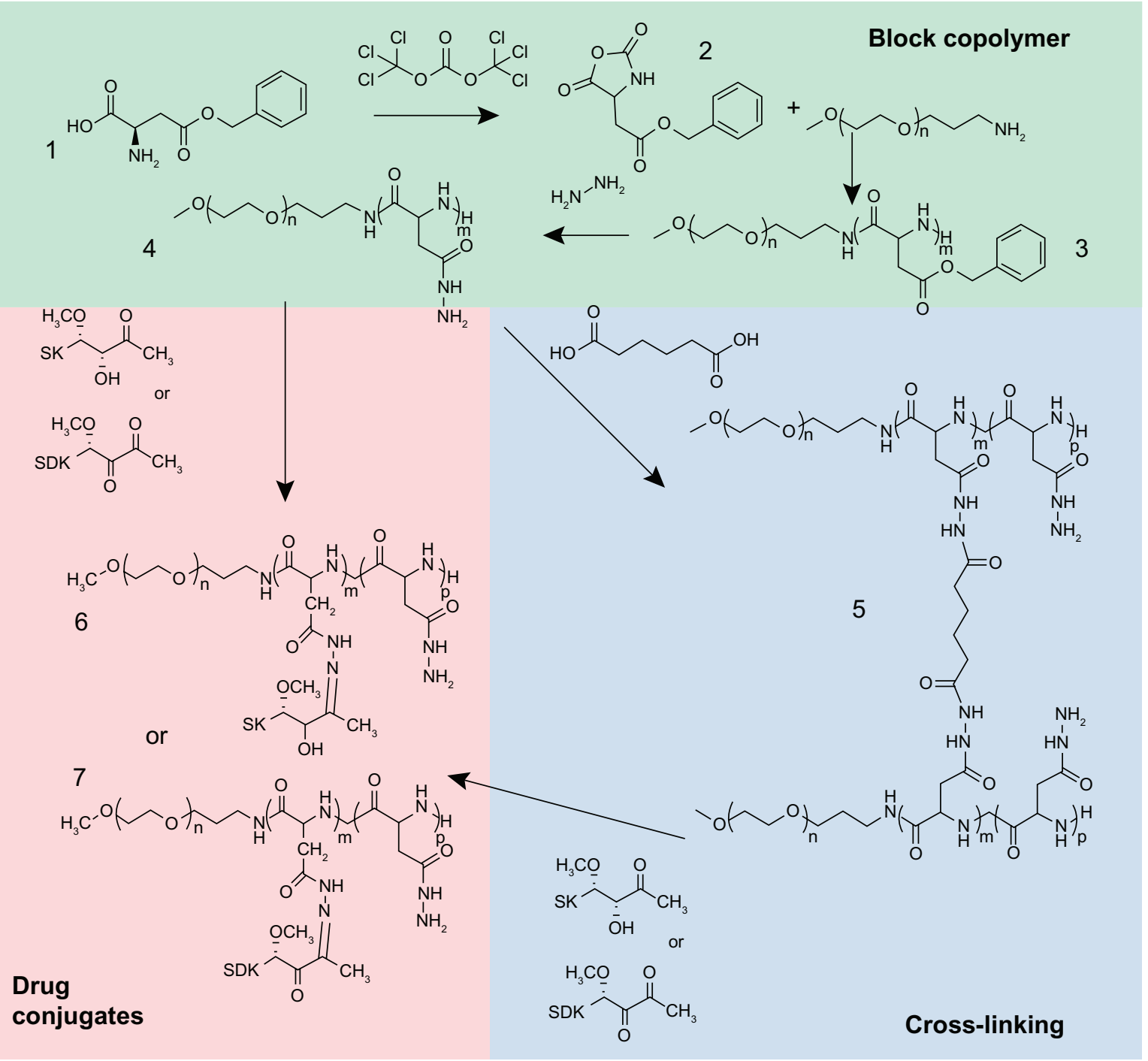

Figure 2 Synthesis of block copolymers, cross-linked block copolymers, and drug conjugates.

block copolymers were isolated from the reaction mixture by precipitation with anhydrous ethyl ether and subsequently freeze drying. PEG-PBLA $(100 \mathrm{mg} / \mathrm{mL})$ was reacted with 20 -fold equivalent hydrazine in DMSO $\left(40^{\circ} \mathrm{C}\right)$ for 1 hour to prepare PEG-p(Asp-Hyd) block copolymers. The materials were collected by ether precipitation and freeze drying. The block copolymer compositions were determined by proton nuclear magnetic resonance spectroscopy ( $\left.{ }^{1} \mathrm{H}-\mathrm{NMR}\right)$, which is described as $\mathrm{X}-\mathrm{Y}$ denoting the molecular weight of PEG $\times 10^{-3}(\mathrm{X})$ and the number of aspartate repeating units $(\mathrm{Y})$, respectively.

\section{Synthesis of self-assembled polymer micelles}

MTM analogs were conjugated to the PEG-p(Asp-Hyd) block copolymers by adding both SK and SDK to the block copolymer at 50 weight \% in DMSO. The reaction was allowed to proceed at room temperature for 72 hours, shaking at $500 \mathrm{rpm}$. The materials were collected and purified by repeating ether precipitation. The drug-polymer conjugates were then re-dissolved in deionized water to form selfassembled micelles. The drug-loaded micelles were collected by freeze drying.

\section{Synthesis of cross-linked polymer micelles}

Cross-linked polymer micelles were created by cross-linking PEG-p(Asp-Hyd) 5-40 with adipic acid. PEG-p(Asp-Hyd) 5-40 was dissolved in DMSO to a final concentration of $100 \mathrm{mg} / \mathrm{mL}$. Adipic acid, NHS, and DIC were combined in DMSO to a mole ratio of 0.5:4:4 times the hydrazide groups of the block copolymer, respectively, and the reaction mixture 
was added to the block copolymer. The cross-linking reaction was conducted for 72 hours, stirring at room temperature. The reaction solution was then dialyzed against DMSO, followed by $50 \%$ DMSO, and finally deionized water. The solution was freeze dried to collect the purified materials. The cross-linked micelles were characterized by gel permeation chromatography (GPC) and ${ }^{1} \mathrm{H}-\mathrm{NMR}$. MTM analogs were conjugated to cross-linked micelles at a 50 weight $\%$ in DMSO, followed by ether precipitation and freeze drying.

\section{Micelle characterization}

Drug loading efficiency in polymer micelles was determined by quantifying the absorbance of MTM analogs at $410 \mathrm{~nm}$. Particle size of the micelles was determined by dynamic light scattering (DLS) on a Zetasizer Nano-ZS (Malvern Instruments Ltd, Worcestershire, UK) equipped with a He-Ne laser light source and $173^{\circ}$ angle scattered light collection configuration.

\section{Drug-release experiments}

Drug-release patterns of the self-assembled or cross-linked polymer micelles were evaluated by using dialysis cassettes (Slide-A-Lyzer G2, MWCO $10 \mathrm{kDa}$ ). Dialysis cassettes containing $2 \mathrm{~mL}$ samples were placed in $4 \mathrm{~L}$ of phosphate buffer solution (10 mM, pH 7.4 or 5.0). The temperature was maintained at $37^{\circ} \mathrm{C}$. An aliquot of $150 \mu \mathrm{L}$ was taken out of each dialysis cassette at the time intervals of $0.0,0.5,1.0$, $3.0,6.0,24.0,48.0$, and 72.0 hours. The aliquots taken at each time point were analyzed by ultraviolet-visible (UV-Vis) absorbance at $410 \mathrm{~nm}$. All experiments were performed in triplicate.

\section{Cell toxicity assays}

The cytotoxic effects of four MTM nanoparticulate formulations were investigated in comparison to free drugs. A549 cells were cultured as specified from ATCC at $37^{\circ} \mathrm{C}$, $5 \% \mathrm{CO}_{2}$. The cells were seeded on a 96 -well plate (5000 cells/ well) and allowed to adhere for 24 hours. After 24 hours, growth media were replaced with fresh media containing samples at differing concentrations. 1\% DMSO (biotechnological grade) was used as a control vehicle to prepare free drug formulations for SK and SDK. Self-assembled and cross-linked micelles were used as nanoparticulate formulations for SK and SDK. The cells were exposed to samples for 72 hours total $(n=8)$. Cell viability was assessed using a resazurin assay that indicates mitochondrial metabolic activity in live cells. A volume of $10 \mu \mathrm{L}$ of a $1 \mathrm{mM}$ resazurin solution in phosphate buffered saline was added to the vehicle- and drug-treated cells at the end of the treatment period. Cell viability was determined 3 hours later by reading the fluorescence at $560 \mathrm{~nm}$ (excitation wavelength)/590 nm (emission wavelength). The fluorescence signals were quantified using a Spectramax M5 plate reader (Molecular Devices, Sunnyvale, CA) equipped with SoftMax ${ }^{\circledR}$ Pro (Molecular Devices) software. Cytotoxicity was determined by calculating the half maximal inhibitory concentration $\left(\mathrm{IC}_{50}\right)$ of each sample.

\section{Results}

\section{Biosynthesis of SK and SDK}

SK and SDK were successfully produced by the $S$. argillaceus $\mathrm{M} 7 \mathrm{~W} 1$, and isolated through an adapted procedure developed previously. ${ }^{15} \mathrm{~S}$. argillaceus cells of the M7W1 mutant strain were plated on R5A agar and allowed to grow until spores were formed. After 2 days, colonies began to appear on the plates; after 3 days, spores began to form; and after 4 days, the majority of the cells were present as spores. Spores from the plate were used to inoculate an initial culture of TSB media. The cells grew quickly in TSB media and were ready to transfer to R5A media after 24 hours. Once transferred to the R5A growth media, the cells were able to produce the drug analogs. Initially the media was adjusted to $\mathrm{pH} 6.85$; however, after the observation of the production, the $\mathrm{pH}$ was adjusted to 7.0. The culture was monitored for the emergence of the MTM analogs using HPLC. After 24 hours, the presence of MTM analogs or precursors was not readily detected. After 48 hours, the MTM analogs began to appear; however, a large population of pre-MTM compounds was still observed. After 72 hours, the MTM compounds were the dominant compounds in the culture so the production was stopped. Much of the population of the MTM analogs was excreted in the culture media, thus the initial step of the isolation was to separate out the cells from the media by the addition of a celite cell-binding resin and subsequent filtration. Once the cells had been removed, the filtrate was passed over a C18 RP silica gel column to collect the compounds of interest. The initial washes of $10 \%$ and $20 \%$ ACN allowed many of the byproducts to be removed from the sample. Further fractionation of the culture on the $\mathrm{C} 18 \mathrm{RP}$ column did not aid in the purification of the individual MTM analogs, so it was decided to elute all the MTM compounds together to minimize sample loss and excessive dilution. The cells removed in the initial filtration step did still contain a substantial amount of SK and SDK, thus to take full advantage of the production of the analogs the cultured cells were covered in $\mathrm{MeOH}$ and sonicated to lyse the cells and release the drugs. 
Cellular debris was removed by again filtering the solution which was then able to be dried, reconstituted in water, and loaded on the C18 RP column. After the same wash and elution steps were performed, a similar solution to the initial cell filtrate was obtained; however, in a smaller quantity. The samples could then be loaded and the individual MTM analogs separated by using semi-preparative HPLC. The individual peaks were collected and analyzed for purity using HPLC-mass spectrometry. Both the SK and SDK products were collected at $>95 \%$ purity through this procedure and were stored at $-20^{\circ} \mathrm{C}$ for future use.

\section{Synthesis of polymer micelles}

${ }^{1} \mathrm{H}-\mathrm{NMR}$ showed that PEG-p(Asp-Hyd) was successfully synthesized as reported previously ${ }^{22}$ and used to prepare self-assembled micelles. For cross-linked micelles, the cross-linking of PEG-p(Asp-Hyd) was confirmed by GPC. The cross-linked micelles had an average molecular weight of $\sim 147 \mathrm{kDa}$, corresponding to an average of roughly 14 block copolymers per cross-linked micelle. NMR was also used to determine the cross-linking percentage. The carboxyl peak of adipic acid at around 12 ppm disappeared, indicating that no unreacted or partially reacted adipic acid was present in the sample. The cross-linking percentage was $12.5 \%$, which was determined by integrating the newly appeared peaks at $2.1 \mathrm{ppm}$ and $1.5 \mathrm{ppm}$ ( $\alpha$ and $\beta \mathrm{CH}_{2}$ groups of adipic acid) with respect to the PEG peak at $3.5 \mathrm{ppm}$.

\section{Characterization of MTM-loaded micelles}

Following the drug conjugation reaction, ether precipitation was used to collect the polymers. Initial precipitations possessed a yellow supernatant corresponding to free drug that was not entrapped in the micelle structures. Ether precipitations were continued until the supernatant remained clear for several precipitations. The polymers maintained a yellowish color, after free drugs were removed, indicating the presence of the MTM analogs. UV-Vis absorption spectrometry at $410 \mathrm{~nm}$ confirmed that both SK and SDK were successfully entrapped in both the self-assembled micelles and cross-linked micelles. Drug loading in the selfassembled micelles was $35.4 \pm 0.3$ and $44.6 \pm 2.2$ weight $\%$ (wt \%) for SK and SDK, respectively. Cross-linked micelles had an average drug loading of $31.3 \pm 0.3$ and $42.7 \pm 5.3$ wt $\%$ for SK and SDK, respectively. DLS showed that the particle size of self-assembled micelles entrapping SK and SDK was $8.36 \pm 3.21$ and $12.19 \pm 2.77 \mathrm{~nm}$. The particle size of cross-linked micelles was $29.56 \pm 4.67 \mathrm{~nm}$ for SK and $30.48 \pm 7.00 \mathrm{~nm}$ for SDK (Figure 3 ).

\section{Drug-release experiments}

A $\mathrm{pH}$ sensitive hydrazone linkage was used to conjugate MTM analogs to the block copolymers to take advantage of the acidic tumor environment. To test $\mathrm{pH}$-responsive drugrelease patterns, MTM nanoparticulate formulations were incubated at pH 7.4 and 5.0. Figure 4 shows that all MTM nanoparticulate formulations released drugs effectively. At pH 7.4, self-assembled micelles entrapped more than 50\% of MTM analogs for 24 hours, which will be stable enough to deliver drugs to tumor tissues following intravenous injections. Drug release from the self-assembled micelles was accelerated at pH 5.0, suggesting that SK and SDK will be released effectively in acidic tumor tissues. Cross-linked micelles appeared to release drugs more quickly at $\mathrm{pH} 7.4$ than self-assembled micelles, yet retained $40 \%$ of drugs for 24 hours. Both SK and SDK were released more rapidly from cross-linked micelles at $\mathrm{pH}$ 5.0.
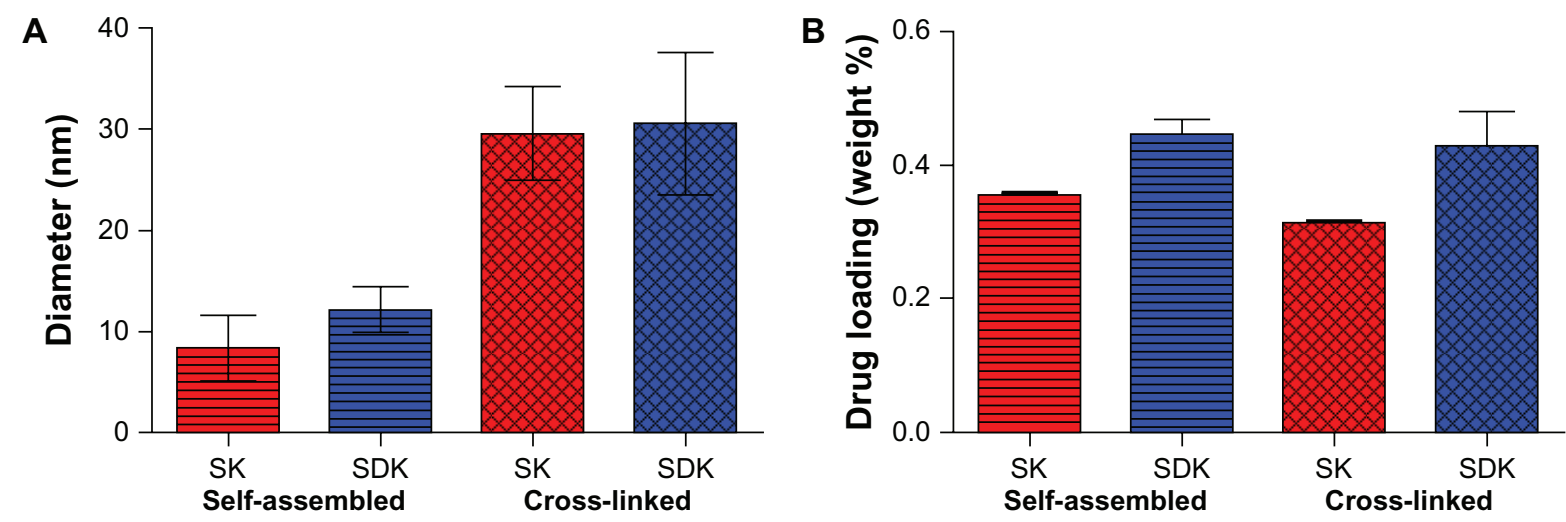

Figure 3 (A) Particle size and (B) drug-entrapment yields.

Abbreviations: SDK, mithramycin SDK; SK, mithramycin SK. 

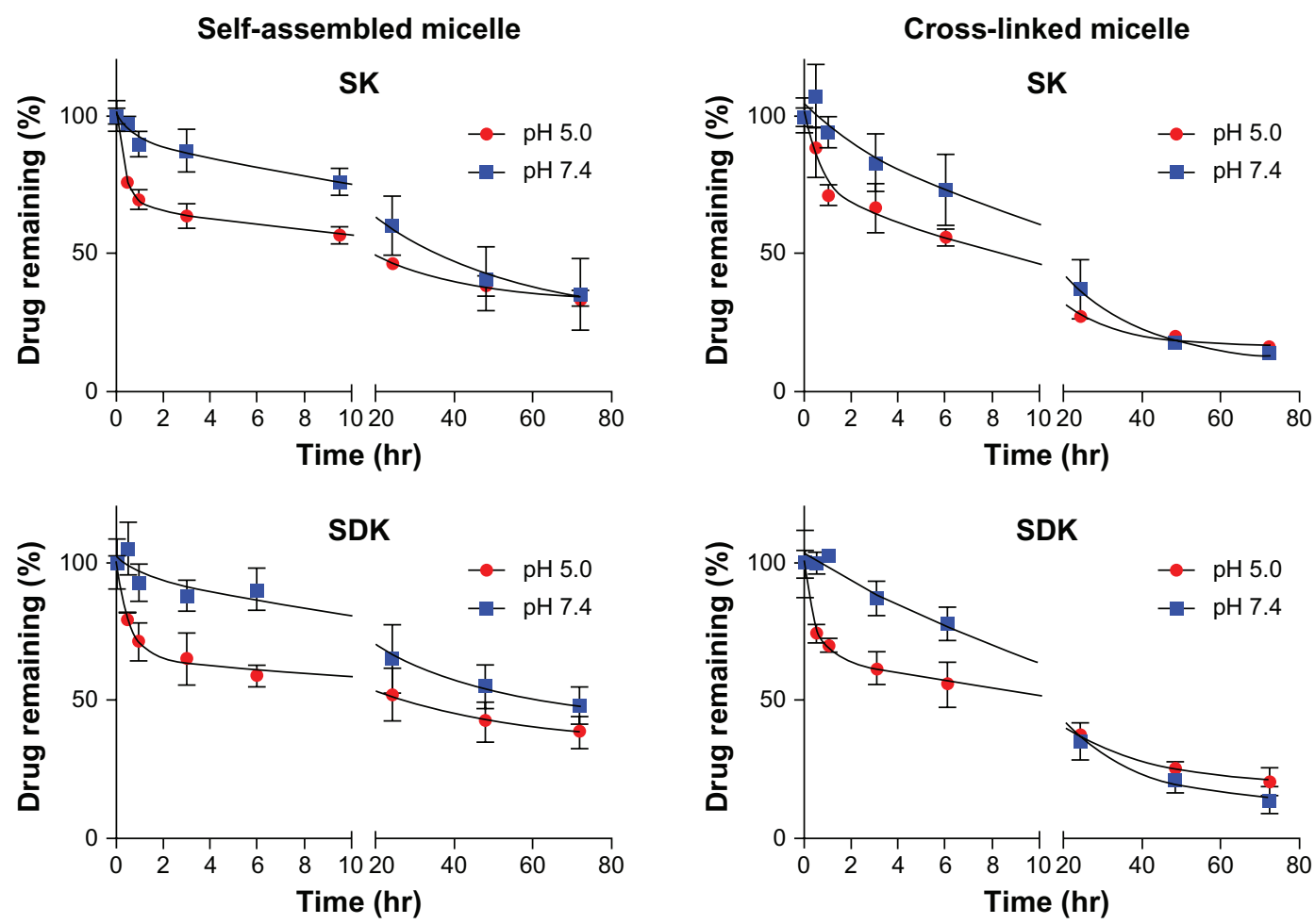

Figure 4 Drug-release patterns from self-assembled and cross-linked micelles at $\mathrm{pH} 7.4$ and 5.0 for 72 hours.

Notes: All data points are the average of three measurements \pm standard deviation; error bars not visible are obstructed by the point.

Abbreviations: SDK, mithramycin SDK; SK, mithramycin SK.

\section{Cell toxicity assays}

Cytotoxicity of MTM nanoparticulate formulations was tested with A549 cells (Figure 5). Free SK and SDK showed $\mathrm{IC}_{50}$ values of $0.163 \pm 0.092 \mu \mathrm{M}$ and $0.093 \pm 0.035 \mu \mathrm{M}$, respectively. $\mathrm{IC}_{50}$ values of self-assembled micelles entrapping MTM analogs were $0.262 \pm 0.087 \mu \mathrm{M}$ and $0.282 \pm 0.131 \mu \mathrm{M}$ for SK and SDK, which were 1.6- and 3.0-fold higher than those of free drugs. Interestingly, crosslinked micelles showed $\mathrm{IC}_{50}$ values of $0.073 \pm 0.015 \mu \mathrm{M}$ and $0.075 \pm 0.009 \mu \mathrm{M}$ for SK and SDK, which were 2.2- and 1.2-fold lower than free drugs (Table 1). No toxicity was observed with block copolymers, self-assembled micelles and cross-linked micelles alone up to $100 \mu \mathrm{M}$ (data not shown).

\section{Discussion}

As mentioned above, MTM has been limited clinically by the severe negative side effects associated with the use of the drug. However, MTM has shown promise with respect to treating neurological diseases, glioblastomas, and other
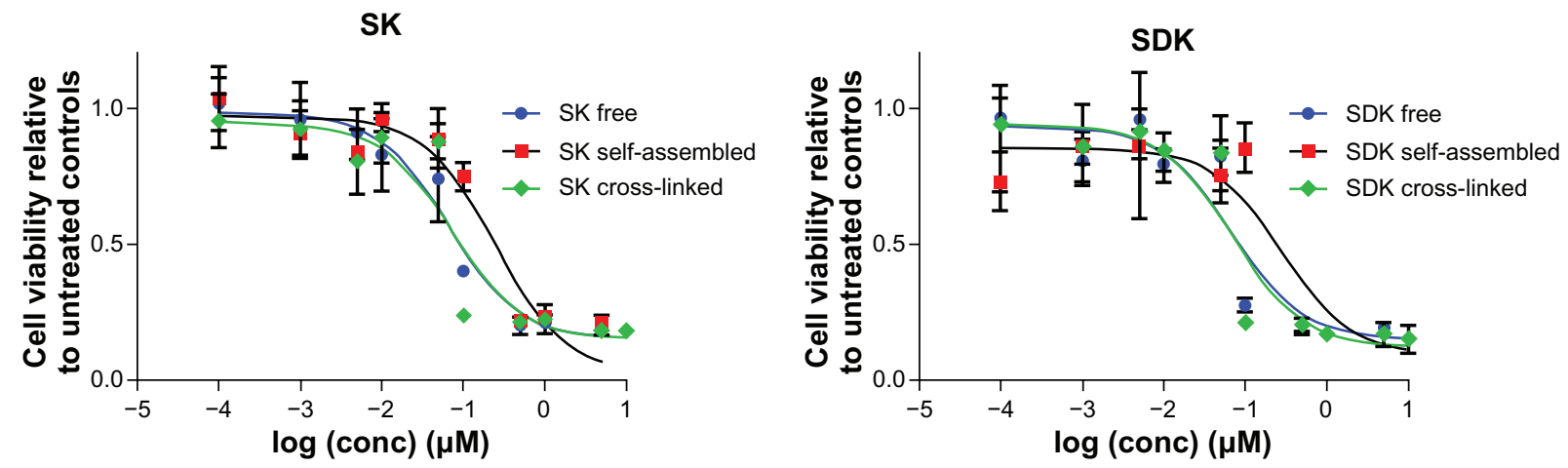

Figure 5 The cytotoxicity of free drug and nanoparticulate formulations for SK and SDK against A549 cell lines.

Notes: All points are the average of four measurements \pm one standard deviation; error bars not visible are obstructed by the point.

Abbreviations: SDK, mithramycin SDK; SK, mithramycin SK. 
Table I A summary of the characteristics and activity of the drug-loaded polymer nanoparticles and that of the free drugs for comparison

\begin{tabular}{|c|c|c|c|c|c|c|}
\hline \multirow{2}{*}{$\begin{array}{l}\text { Drug } \\
\text { formulation }\end{array}$} & \multicolumn{3}{|l|}{ SK } & \multicolumn{3}{|l|}{ SDK } \\
\hline & Free & $\begin{array}{l}\text { Self-assembled } \\
\text { micelles }\end{array}$ & $\begin{array}{l}\text { Cross-linked } \\
\text { micelles }\end{array}$ & Free & $\begin{array}{l}\text { Self-assembled } \\
\text { micelles }\end{array}$ & $\begin{array}{l}\text { Cross-linked } \\
\text { micelles }\end{array}$ \\
\hline $\mathrm{IC}_{50}(\mathrm{nM})$ & $163 \pm 92$ & $262 \pm 87$ & $73 \pm 15$ & $93 \pm 36$ & $282 \pm 131$ & $75 \pm 9$ \\
\hline Relative efficacy to free drug & 1.00 & 0.62 & 2.23 & 1.00 & 0.33 & 1.24 \\
\hline
\end{tabular}

Abbreviations: SDK, mithramycin SDK; SK, mithramycin SK.

tumors in addition to showing the ability to inhibit the multidrug resistance gene, MDR1, in which smaller, less toxic doses are required. ${ }^{32-37}$ Recently, MTM was identified as the lead compound against the Friend leukemia virus integration 1 (EWS-FLI1) transcription factor. ${ }^{38}$ EWS-FLI1 is believed to be responsible for malignant transformation and progression of Ewing sarcoma family tumors and was widely regarded as undruggable because it is a transcription factor. ${ }^{38} \mathrm{~A}$ high-throughput screening of over 50,000 compounds identified MTM as the top candidate from the screen and greatly reduced and inhibited tumor volume in Ewing sarcoma family tumors in mouse xenograft models, and it is planned to reinstitute clinical trials for this application of MTM. ${ }^{38}$ Additionally, a combinational approach of MTM with betulinic acid to treat pancreatic cancer showed the ability to synergistically treat the cancer with a nontoxic dose of the drugs to produce less discernible side effects. ${ }^{35}$ As new treatments and uses for MTM continue to be uncovered, the development and optimization of a compatible drugdelivery system will be necessary.

The potential of MTM has been enhanced by the discovery of two new analogs produced through engineering of the MTM biosynthetic pathway. These analogs, SK and SDK, are a result of the inactivation of the $m t m W$ gene and have shown improved activity compared with that of the native MTM. The biosynthesis and isolation of the SK and SDK analogs are pivotal as larger quantities of the drug will be required for testing and eventually clinical use. SK and SDK are produced alongside two other compounds, a demycarosyl-MTM SK and MTM SA (SA). The SA analog shows decreased anticancer activity and the demycarosylMTM SK shows activity similar to that of the native MTM, thus neither are the preferred products of the mutant strain. Initially, a culture $\mathrm{pH}$ of 6.85 was used for the production of the drugs. These conditions allowed for good production of the SK, but a large amount of SA and very little SDK were also being observed. The formation of SK, SDK, and SA are a result of the spontaneous rearrangement of the immediate product MTM DK, resulting from the MtmOIV reaction of the biosynthetic pathway. ${ }^{15,16}$ It has been shown by in-vitro work that the major product of the conversion of premithramycin B by MtmOIV can be shifted to SDK at a $\mathrm{pH}$ of $8.25 .{ }^{39} \mathrm{In}$ an effort to improve the production of SDK from the culture, the $\mathrm{pH}$ of the media was adjusted to 7.0. It was hoped that the adjustment of the $\mathrm{pH}$, although small, would still allow optimal growth of the M7W1 mutant strain and production of the MTM analogs, but that the production yield of SDK would be improved. Increasing the $\mathrm{pH}$ of the growth media to 7.0 confirmed the hypothesis and increased the production yield of SDK. Increasing the $\mathrm{pH}$ also did not appear to adversely affect the production of SK. This will be critical as the production of the analogs is scaled up to meet the clinical demands.

As the drive for more specific and efficient chemotherapeutic options continues, delivery systems will undoubtedly play a pivotal role in their development and advancement. To that end, these novel MTM formulations still need to resolve issues such as low tumor accumulation, poor bioavailability, and short plasma retention time to be further explored clinically. Drug-delivery systems are expected to address these concerns by delivering MTM preferentially to tumors. Liposomal formulations have been previously tested, but the release of MTM analogs from liposomes has proved to be difficult. ${ }^{40}$ Studies suggest that polymer micelles will offer an alternative platform for the delivery of MTM, not only delivering the drug to tumors but also controlling the drugrelease patterns efficiently. It has been demonstrated that block copolymers offer a versatile platform in this direction. In this study, MTM analogs (SK and SDK) were entrapped in polymer micelles through an acid-labile hydrazone conjugation between drug molecules and micelle-forming block copolymers. Prepared MTM nanoparticulate formulations appeared to combine the favorable characteristics of drugdelivery systems with the ability to entrap drugs at high yields and to control the drug release at the area of interest.

SK and SDK have one and two ketone groups on the side chain. These ketone groups offer a prime location for the conjugation of the drugs to the PEG-p(Asp-Hyd) block 
copolymers through the hydrazone bond. Self-assembled micelles showed higher drug loading of SDK (44.6 wt \%) than SK (35.4 wt \%), suggesting that the second ketone group of the SDK side chain could facilitate drug loading rather than saturating the drug-binding sites. The particle size of selfassembled micelles was 44\% larger with SDK (12.19 nm) than SK $(8.36 \mathrm{~nm})$, which is consistent with the increased SDK loading in micelles. The average particle size of crosslinked micelles was $26.79 \mathrm{~nm}$ in the absence of drugs. Crosslinked micelles were 29.56 and $30.48 \mathrm{~nm}$ after entrapping SK and SDK, respectively. Even though the particle size was retained, cross-linked micelles entrapped $31.3 \mathrm{wt} \% \mathrm{SK}$ and 42.7 wt \% SDK, which were comparable to the amount of drugs that self-assembled micelles entrapped.

Again, an acid labile hydrazone bond was used to conjugate the drugs to the block copolymers so the release of the drugs from the nanoformulations was investigated at both the physiological pH of 7.4 and an intracellular endosome or lysosome $\mathrm{pH}$ of 5.0. Effective drug release was observed in all MTM nanoparticulate formulations tested improving on previous attempts of MTM delivery with liposomes. MTM analogs were released slowly from polymer micelles at $\mathrm{pH} 7.4$ regardless of formulations. The drug release was accelerated at pH 5.0 (Figure 4), which is advantageous to release drugs in acidic tumor tissues. After 72 hours, however, the amount of drugs released was similar at both $\mathrm{pH}$ values. MTM nanoparticulate formulations are expected to minimize drug leakage in the blood and maximize therapeutic efficacy by releasing drugs in tumor tissues rapidly.

MTM nanoparticulate formulations were shown to be effective to suppress cancer cell growth, indicating that MTM analogs were delivered in a biologically active form. Self-assembled micelles entrapping MTM analogs showed lower cytotoxic effects than free drugs as expected, but interestingly, cross-linked micelles enhanced drug efficacy up to 2.2-fold (Table 1). Although further investigation is necessary to elucidate the mechanism behind this intriguing phenomenon, the results of this study suggest that drug release in the early stage would not play a crucial role in determining cellular response to MTM analogs as all nanoparticulate formulations released a similar amount of drugs between 1 and 3 hours. Drug release at $\mathrm{pH} 7.4$ rather than 5.0 appeared to be the major factor that determines cytotoxicity. MTM analogs were effective to suppress cancer cell growth in order of cross-linked SK micelles, cross-linked SDK micelles, self-assembled SK micelles, and self-assembled SDK micelles, which is consistent with the drug-release patterns at $\mathrm{pH} 7.4$, whereas all nanoparticulate formulations released drugs similarly at $\mathrm{pH}$ 5.0, which does not explain differential cytotoxicity observed in A549. Therefore, it is more reasonable to assume that drugs released from micelles at $\mathrm{pH} 7.4$ comprise the major drug population that involved in cell killing, while intracellular drug release $(\mathrm{pH} 5.0)$ has less effect on overall cytotoxicity of MTM nanoparticulate formulations. However, detailed intracellular mechanisms on how cells respond to MTM nanoparticulate formulations (eg, particle size and intracellular drug distribution) should be investigated further in the future.

Cross-linked micelles had a $12.5 \%$ cross-linking yield, which was high enough to tether block copolymer chains before and after entrapping drugs without causing agglomeration between particles. In spite of the high cross-linking yield, cross-linked micelles entrapped and released MTM analogs effectively. Importantly cross-linked micelles released drugs more quickly than self-assembled micelles, implicating that MTM analogs could be entrapped in the vicinity rather than at the center of the micelle core. The rapid drug release from cross-linked micelles can be also attributed to the rigid environment in the cross-linked core of the micelles. Cross-linking the core could allow for a more porous and less tightly packed core in the micelles so that drug molecules can maneuver more easily out of the core. In contrast, self-assembled micelles have a flexible core that can continue to pull block copolymers to keep the particle stable as drugs are released. This may explain why drug release from self-assembled micelles was slower than cross-linked micelles.

Cytotoxic effects of MTM nanoparticulate formulations revealed an interesting phenomenon that can be used to design potent drug-delivery systems. Unlike self-assembled micelles that decreased the toxicity of MTM analogs, crosslinked micelles showed a higher potency against A549 cancer cells than free drugs. The exact mechanism and reason for the improved efficacy is yet to be confirmed, although the more stable cross-linked micelles could aid in the drug's persistence in the cells, allowing more drugs to interact with the therapeutic targets in the cell. In addition to the known mechanism of the MTM family of compounds in which they bind the GC rich DNA present in Sp family of promoters, the expression of the gene encoding for a multidrug resistance efflux pump, MDR1, has also been shown to be inhibited by MTM and some of it derivatives. ${ }^{36,37}$ This would make the MTM nanoparticulate formulations excellent candidates for combinational therapy attacking hard-to-treat, multidrugresistant cancer cell lines. Future studies will explore 
whether these results translate in vivo and dig deeper into the reasoning behind the improved activity.

\section{Conclusion}

Nanoparticulate formulations for controlled delivery of MTM analogs, SK and SDK, were prepared by using self-assembled or cross-linked polymer micelles from PEG-p(Asp-Hyd) block copolymers to which drugs were conjugated through acid-labile hydrazone bonds. With recent interest in MTM for several novel treatment approaches, a compatible delivery system with ability to selectively deliver the drug to the area of interest would be advantageous. Both the SK and SDK analogs offer higher anticancer activity compared with MTM and thus were investigated with respect to a compatible delivery system. SK and SDK were successfully loaded into polymer micelles and demonstrated a $\mathrm{pH}$-dependent release profile, accelerating drug release at $\mathrm{pH}$ 5.0. By cross-linking the micelles, the size of the particles was increased without significantly affecting the drug loading. Both the selfassembled and cross-linked structures were able to deliver the drugs in the biologically active form and efficiently kill the human nonsmall cell lung cancer cell line (A549). The crosslinked micelles interestingly improved the cytotoxicity of MTM analogs, even compared with the free drugs. The ability to prepare delivery platforms without losing any biological activity will streamline the development of therapeutic options, and these results conclude that nanoparticulate formulations offer prime candidates to pave the path for SK, SDK, and other MTM analogs to be explored further with animal models and potentially in the clinical field.

\section{Acknowledgments}

This research was supported in part by the National Institutes of Health (grant CA 091901 to JR) and the Kentucky Lung Cancer Research Program (to YB). DS acknowledges financial support from a NCI-CNTC postdoctoral traineeship, and the project described was supported by grant number 5R25CA153954 from the National Cancer Institute. The content is solely the responsibility of the authors and does not necessarily represent the official views of the National Cancer Institute or the National Institutes of Health.

\section{Disclosure}

The authors report no conflicts of interest in the work. The University of Kentucky has determined that it has a legal ownership interest in this intellectual property based on material use of university resources and the appropriate protection for this intellectual property will be pursued.

\section{References}

1. Miller DM, Polansky DA, Thomas SD, et al. Mithramycin selectively inhibits transcription of G-C containing DNA. Am J Med Sci. 1987; 294(5):388-394.

2. Sastry M, Fiala R, Patel DJ. Solution structure of mithramycin dimers bound to partially overlapping sites on DNA. J Mol Biol. 1995;251(5): 674-689.

3. Sastry M, Patel DJ. Solution structure of the mithramycin dimer-DNA complex. Biochemistry. 1993;32(26):6588-6604.

4. Blume SW, Snyder RC, Ray R, Thomas S, Koller CA, Miller DM. Mithramycin inhibits SP1 binding and selectively inhibits transcriptional activity of the dihydrofolate reductase gene in vitro and in vivo. J Clin Invest. 1991;88(5):1613-1621.

5. Snyder RC, Ray R, Blume S, Miller DM. Mithramycin blocks transcriptional initiation of the c-myc $\mathrm{P} 1$ and $\mathrm{P} 2$ promoters. Biochemistry. 1991;30(17):4290-4297.

6. Safe S, Abdelrahim M. Sp transcription factor family and its role in cancer. Eur J Cancer. 2005;41(16):2438-2448.

7. Abdelrahim M, Smith R 3rd, Burghardt R, Safe S. Role of Sp proteins in regulation of vascular endothelial growth factor expression and proliferation of pancreatic cancer cells. Cancer Res. 2004;64(18): 6740-6749.

8. Black AR, Black JD, Azizkhan-Clifford J. Sp1 and kruppel-like factor family of transcription factors in cell growth regulation and cancer. J Cell Physiol. 2001;188(2):143-160.

9. Kennedy BJ. Metabolic and toxic effects of mithramycin during tumor therapy. Am J Med. 1970;49(4):494-503.

10. Brown JH, Kennedy BJ. Mithramycin in the treatment of disseminated testicular neoplasms. N Engl J Med. 1965;272:111-118.

11. Koller CA, Miller DM. Preliminary observations on the therapy of the myeloid blast phase of chronic granulocytic leukemia with plicamycin and hydroxyurea. N Engl J Med. 1986;315(23):1433-1438.

12. Ryan WG. Mithramycin for Paget's disease of bone. $N$ Engl J Med. 1970;283(21):1171.

13. Ryan WG, Schwartz TB, Northrop G. Experiences in the treatment of Paget's disease of bone with mithramycin. JAMA. 1970;213(7): 1153-1157.

14. Remsing LL, Garcia-Bernardo J, Gonzalez A, et al. Ketopremithramycins and ketomithramycins, four new aureolic acid-type compounds obtained upon inactivation of two genes involved in the biosynthesis of the deoxysugar moieties of the antitumor drug mithramycin by Streptomyces argillaceus, reveal novel insights into post-PKS tailoring steps of the mithramycin biosynthetic pathway. J Am Chem Soc. 2002; 124(8):1606-1614.

15. Albertini V, Jain A, Vignati S, et al. Novel GC-rich DNA-binding compound produced by a genetically engineered mutant of the mithramycin producer Streptomyces argillaceus exhibits improved transcriptional repressor activity: implications for cancer therapy. Nucleic Acids Res. 2006;34(6):1721-1734.

16. Remsing LL, González AM, Nur-e-Alam M, et al. Mithramycin SK, a novel antitumor drug with improved therapeutic index, mithramycin SA, and demycarosyl-mithramycin SK: three new products generated in the mithramycin producer Streptomyces argillaceus through combinatorial biosynthesis. J Am Chem Soc. 2003;125(19):5745-5753.

17. Takakura Y, Fujita T, Hashida M, Sezaki H. Disposition characteristics of macromolecules in tumor-bearing mice. Pharm Res. 1990;7(4): 339-346.

18. van Vlerken L, Vyas T, Amiji M. Poly(ethylene glycol)-modified Nanocarriers for tumor-targeted and intracellular delivery. Pharm Res. 2007;24(8):1405-1414.

19. Smart T, Lomas H, Massignani M, Flores-Merino MV, Perez LR, Battaglia G. Block copolymer nanostructures. Nano Today. 2008; 3(3-4):38-46.

20. Bae Y, Kataoka K. Intelligent polymeric micelles from functional poly(ethylene glycol)-poly(amino acid) block copolymers. Adv Drug Deliv Rev. 2009;61(10):768-784. 
21. Chen H, Kim S, Li L, Wang S, Park K, Cheng JX. Release of hydrophobic molecules from polymer micelles into cell membranes revealed by Forster resonance energy transfer imaging. Proc Natl Acad Sci U S A. 2008;105(18):6596-6601.

22. Lee HJ, Bae Y. Cross-linked nanoassemblies from poly(ethylene glycol)-poly(aspartate) block copolymers as stable supramolecular templates for particulate drug delivery. Biomacromolecules. 2011; 12(7):2686-2696.

23. Becker ML, Remsen EE, Pan D, Wooley KL. Peptide-derivatized shellcross-linked nanoparticles. 1. Synthesis and characterization. Bioconjug Chem. 2004;15(4):699-709.

24. West KR, Otto S. Reversible covalent chemistry in drug delivery. Curr Drug Discov Technol. 2005;2(3):123-160.

25. Jones AT, Gumbleton M, Duncan R. Understanding endocytic pathways and intracellular trafficking: a prerequisite for effective design of advanced drug delivery systems. Adv Drug Deliv Rev. 2003;55(11): 1353-1357.

26. Vander Heiden MG, Cantley LC, Thompson CB. Understanding the Warburg effect: the metabolic requirements of cell proliferation. Science. 2009;324(5930):1029-1033.

27. Gillies RJ, Robey I, Gatenby RA. Causes and consequences of increased glucose metabolism of cancers. J Nucl Med. 2008;49 Suppl 2: $4 \mathrm{~S}-42 \mathrm{~S}$.

28. Bae Y, Nishiyama N, Kataoka K. In vivo antitumor activity of the folate-conjugated $\mathrm{pH}$-sensitive polymeric micelle selectively releasing adriamycin in the intracellular acidic compartments. Bioconjug Chem. 2007;18(4):1131-1139.

29. Howard MD, Ponta A, Eckman A, Jay M, Bae Y. Polymer micelles with hydrazone-ester dual linkers for tunable release of dexamethasone. Pharm Res. 2011;28(10):2435-2446.

30. Ponta A, Bae Y. PEG-poly(amino acid) block copolymer micelles for tunable drug release. Pharm Res. 2010;27(11):2330-2342.

31. Alani AW, Bae Y, Rao DA, Kwon GS. Polymeric micelles for the pH-dependent controlled, continuous low dose release of paclitaxel. Biomaterials. 2010;31(7):1765-1772.
32. Jin H-J, Kanthasamy A, Anantharam V, Rana A, Kanthasamy AG. Transcriptional regulation of pro-apoptotic protein kinase $\mathrm{C} \delta$ : implications for oxidative stress-induced neuronal cell death. J. Biol Chem. 2011;286:19840-19859.

33. Seznec J, Silkenstedt B, Naumann U. Therapeutic effects of the Sp1 inhibitor mithramycin A in glioblastoma. J Neurooncol. 2011;101: 365-377.

34. Sleiman SF, Langley BC, Basso M, et al. Mithramycin is a gene-selective Sp1 inhibitor that identifies a biological intersection between cancer and neurodegeneration. J Neurosci. 2011;31:6858-6870.

35. Gao Y, Jia Z, Kong X, et al. Combining betulinic acid and mithramycin A effectively suppresses pancreatic cancer by inhibiting proliferation, invasion, and angiogenesis. Cancer Res. 2011;71:5182-5193.

36. Tagashira M, Kitagawa T, Isonishi S, Okamoto A, Ochiai K, Ohtake Y. Mithramycin represses MDR1 gene expression in vitro, modulating multidrug resistance. Biol Pharm Bull. 2000;23(8):926-929.

37. Tagashira M, Kitagawa T, Nozato N, et al. Two novel C-glycosides of aureolic acid repress transcription of the MDR1 gene. Chem Pharm Bull (Tokyo). 2000;48(4):575-578.

38. Grohar PJ, Woldemichael GM, Griffin LB, et al. Identification of an Inhibitor of the EWS-FLI1 oncogenic transcription factor by highthroughput screening. J Natl Cancer Inst. 2011;103:962-978.

39. Gibson M, Nur-e-alam M, Lipata F, Oliveira MA, Rohr J. Characterization of kinetics and products of the Baeyer-Villiger oxygenase MtmOIV, the key enzyme of the biosynthetic pathway toward the natural product anticancer drug mithramycin from Streptomyces argillaceus. JAm Chem Soc. 2005;127(50):17594-17595.

40. Frezard F, Garnier-Suillerot A, Demicheli C. Encapsulation of mithramycin in liposomes in response to a transmembrane gradient of calcium ions. J Incl Phenom Macrocycl Chem. 1997;28(1):51-62.
International Journal of Nanomedicine

\section{Publish your work in this journal}

The International Journal of Nanomedicine is an international, peerreviewed journal focusing on the application of nanotechnology in diagnostics, therapeutics, and drug delivery systems throughout the biomedical field. This journal is indexed on PubMed Central,

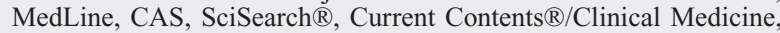

\section{Dovepress}

Journal Citation Reports/Science Edition, EMBase, Scopus and the Elsevier Bibliographic databases. The manuscript management system is completely online and includes a very quick and fair peer-review system, which is all easy to use. Visit http://www.dovepress.com/ testimonials.php to read real quotes from published authors. 